\title{
MINLP optimization of the single-storey industrial building steel structure
}

\author{
T. Žula, U. Klanšek \& S. Kravanja \\ University of Maribor, Faculty of Civil Engineering, Maribor, Slovenia
}

\begin{abstract}
In this paper the optimization of the single-storey industrial building steel structure is presented. The structure consists of the main portal frames, which are mutually connected with the purlins. All structural elements are proposed to be built up of standard hot rolled I sections. The structural optimization is performed by the Mixed-Integer Non-linear programming approach, MINLP. The MINLP performs a discrete topology and standard dimension optimization, while continuous parameters are simultaneously calculated inside the continuous space. Since the discrete/continuous optimization problem of steel frames is non-convex and highly non-linear, the Modified Outer-Approximation/EqualityRelaxation (OA/ER) algorithm has been used for the optimization. Alongside the optimal structure mass, the optimal topology (an optimal number of main portal frames and purlins) as well as all standard cross-section sizes have been obtained. The paper includes the theoretical basis and a practical example with the results of the optimization.
\end{abstract}

\section{Introduction}

Single-storey frame structures are extensively used for industrial, leisure and commercial buildings. In order to obtain efficient frame designs, researchers have introduced various optimization techniques, appropriate either for the continuous or the discrete optimization. O'Brien and Dixon [1] have proposed a linear programming approach for the optimal design of pitched roof frames. Guerlement et al. [2] have introduced a practical method for single-storey steel structures, based on a discrete minimum weight design and Eurocode 3 [3] design constraints. Recently, Saka [4] has considered an optimum design of pitched roof steel frames with haunched rafters by using a genetic algorithm. 
One of the latest researches reported in this field is the work of Hernández et al. [5], where authors have considered minimum weight design of steel portal frames with software developed for structural optimization.

This paper deals with topology and standard dimension optimization of unbraced single-storey industrial building steel structures. The optimization of portal frames is performed by the Mixed-Integer Nonlinear Programming, MINLP. The MINLP is a combined discrete and continuous optimization technique. In this way, the MINLP performs the discrete topology (i.e. the number of frames and purlins) and standard dimension (i.e. the standard crosssection sizes of columns, beams and purlins) optimization simultaneously with the continuous optimization of parameters (e.g. a structure mass, internal forces, deflections, etc.).

The MINLP discrete/continuous optimization problems of frames are in most cases comprehensive, non-convex and highly non-linear. The optimization is proposed to be performed through three steps. The first one includes the generation of a mechanical superstructure of different topology and standard dimension alternatives, the second one involves the development of an MINLP model formulation and the last one consists of a solution for the defined MINLP optimization problem.

The objective of the optimization is to minimize the mass of the single-storey industrial building. The mass objective function is subjected to the set of the equality and inequality constraints known from the structural analysis and dimensioning. The dimensioning of steel members is performed in accordance with Eurocode 3.

The Modified Outer-Approximation/Equality-Relaxation algorithm is used to perform the optimization, see Kravanja and Grossmann [6], Kravanja et al. [7-8].

The two-phase MINLP optimization is proposed. It starts with the topology optimization, while standard dimensions are relaxed temporary into continuous parameters. When the optimal topology is found, standard dimensions of crosssections are re-established and standard dimension optimization of beams, columns and purlins is then continued until the optimal solution is found.

\section{Single-storey industrial building}

The paper presents the topology and standard dimension optimization of unbraced rigid single-storey industrial building steel structures, see Figure 1. Columns, beams and purlins are proposed to be built up of standard hot rolled steel I sections.

The considered portal frame structures are analyzed under the combined effects of the self-weight of frame members, uniformly distributed surface variable load (snow and wind), concentrated horizontal variable load (wind) and an initial frame imperfection. The purlins are designed to transfer permanent load (self-weight of purlins and weight of roof) and variable load (snow and wind) to frame structure. Internal forces are calculated by the elastic first-order analysis. The dimensioning of steel members is performed in accordance with Eurocode 3 
for the conditions of both ultimate limit state (ULS) and serviceability limit state (SLS).

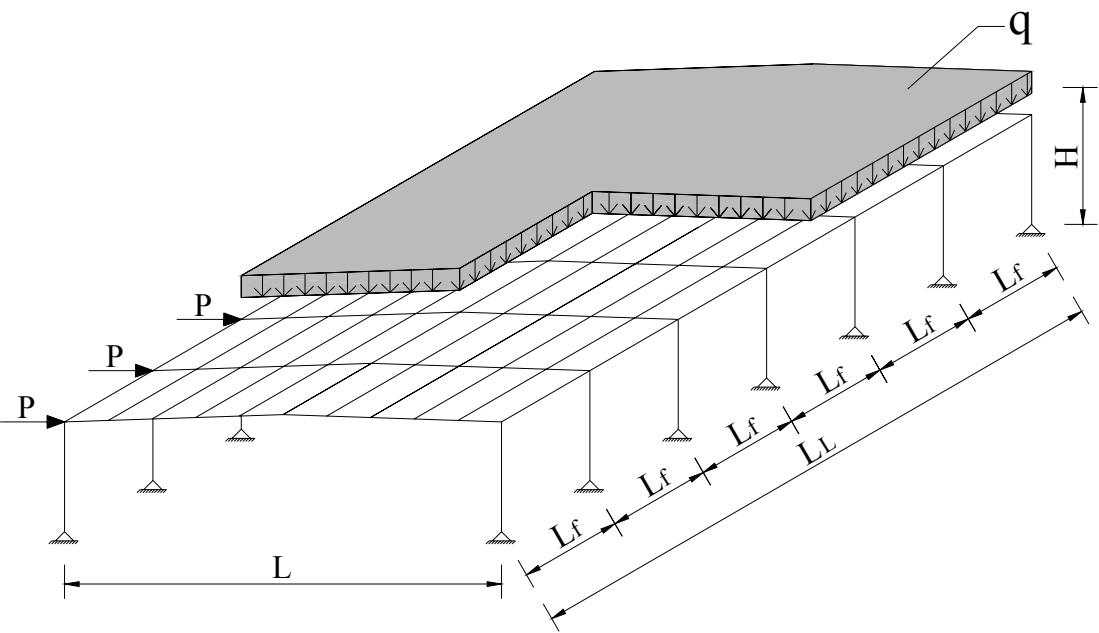

Figure 1: $\quad$ Single-storey industrial building.

When the ultimate limit state of structural members is considered, the elements are checked for axial resistance, shear resistance, bending moment resistance, interaction between bending moment and axial force, interaction between axial compression/buckling and buckling resistance moment.

Considering the serviceability limit state, the vertical deflections of beams and purlins are calculated by the force method. The total deflection $\delta_{\max }$ subjected to the overall load and the deflections $\delta_{2}$ subjected to the variable imposed load are calculated to be smaller than limited maximum values: span/200 and span/250, respectively. The horizontal deflections $\Delta$ are also checked for the recommended limits: the relative horizontal deflection should be smaller then the height/150 of the portal frame.

\section{MINLP model formulation for mechanical superstructures}

It is assumed that a general nonconvex and nonlinear discrete/continuous optimization problem can be formulated as an MINLP problem (MINLP-G) in the form:

$$
\begin{array}{r}
\min z=\boldsymbol{c}^{T} \boldsymbol{y}+f(\boldsymbol{x}) \\
\text { s.t. } \quad \boldsymbol{h}(\boldsymbol{x})=\boldsymbol{0} \\
\boldsymbol{g}(\boldsymbol{x}) \leq \boldsymbol{0} \\
\boldsymbol{B} \boldsymbol{y}+\boldsymbol{C} \boldsymbol{x} \leq \boldsymbol{b}
\end{array}
$$




$$
\begin{gathered}
x \in X=\left\{\boldsymbol{x} \in R^{n}: \boldsymbol{x}^{\mathrm{LO}} \leq \boldsymbol{x} \leq \boldsymbol{x}^{\mathrm{UP}}\right\} \\
\boldsymbol{y} \in Y=\{0,1\}^{m}
\end{gathered}
$$

where $\boldsymbol{x}$ is a vector of continuous variables specified in the compact set $X$ and $\boldsymbol{y}$ is a vector of discrete, mostly binary $0-1$ variables. Functions $f(\boldsymbol{x}), \boldsymbol{h}(\boldsymbol{x})$ and $\boldsymbol{g}(\boldsymbol{x})$ are nonlinear functions involved in the objective function $z$, equality and inequality constraints, respectively. Finally, $\boldsymbol{B} \boldsymbol{y}+\boldsymbol{C} \boldsymbol{x} \leq \boldsymbol{b}$ represents a subset of mixed linear equality/inequality constraints.

The above general MINLP model formulation has been adapted for the synthesis of mechanical superstructures (MINLP-SMS). The resulted formulation that is more specific, particularly in variables and constraints. It can be used also for the modelling the steel frames. It is given in the following form:

$$
\begin{gathered}
\min \quad z=\boldsymbol{c}^{T} \boldsymbol{y}+f(\boldsymbol{x}) \\
\text { s.t. } \quad \boldsymbol{h}(\boldsymbol{x})=\boldsymbol{0} \\
\boldsymbol{g}(\boldsymbol{x}) \leq \boldsymbol{0} \\
\boldsymbol{A}(\boldsymbol{x}) \leq \boldsymbol{a} \\
\boldsymbol{E} \boldsymbol{y} \leq \boldsymbol{e} \\
\boldsymbol{D}^{e}+\boldsymbol{R}(\boldsymbol{x}) \leq \boldsymbol{r} \\
\boldsymbol{K} \boldsymbol{y}^{e}+\boldsymbol{L}\left(\boldsymbol{d}^{c n}\right) \leq \boldsymbol{k} \\
\boldsymbol{P} \boldsymbol{y}+\boldsymbol{S}\left(\boldsymbol{d}^{s t}\right) \leq \boldsymbol{s} \\
\boldsymbol{x} \in X=\left\{\boldsymbol{x} \in R{ }^{n}: \boldsymbol{x}^{\mathrm{LO}} \leq \boldsymbol{x} \leq \boldsymbol{x}^{\mathrm{UP}}\right\} \\
\boldsymbol{y} \in Y=\{0,1\}^{m}
\end{gathered}
$$

The MINLP model formulation for mechanical superstructures is proposed to be described as follows:

- Included are continuous variables $\boldsymbol{x}=\{\boldsymbol{d}, \boldsymbol{p}\}$ and discrete binary variables $\boldsymbol{y}=\left\{\boldsymbol{y}^{e}, \boldsymbol{y}^{s t}\right\}$. Continuous variables are partitioned into design variables $\boldsymbol{d}=\left\{\boldsymbol{d}^{c n}\right.$, $\left.\boldsymbol{d}^{\text {st }}\right\}$ and into performance (nondesign) variables $\boldsymbol{p}$, where subvectors $\boldsymbol{d}^{c n}$ and $\boldsymbol{d}^{\text {st }}$ stand for continuous and standard dimensions, respectively. Subvectors of binary variables $\boldsymbol{y}^{e}$ and $\boldsymbol{y}^{s t}$ denote the potential existence of structural elements inside the superstructure (the topology determination) and the potential selection of standard dimension alternatives, respectively.

- The mass or economical objective function $z$ involves fixed mass or cost charges in the linear term $\boldsymbol{c}^{\mathrm{T}} \boldsymbol{y}$ and dimension dependant mass or costs in the term $f(\boldsymbol{x})$.

- Parameter nonlinear and linear constraints $\boldsymbol{h}(\boldsymbol{x})=\boldsymbol{0}, \boldsymbol{g}(\boldsymbol{x}) \leq \boldsymbol{0}$ and $\boldsymbol{A}(\boldsymbol{x}) \leq \boldsymbol{a}$ represent the rigorous system of the design, loading, stress, deflection, stability, etc. constraints known from the structural analysis.

- Integer linear constraints $\boldsymbol{E} \boldsymbol{y} \leq \boldsymbol{e}$ are proposed to describe relations between binary variables. 
- Mixed linear constraints $\boldsymbol{D y}^{e}+\boldsymbol{R}(\boldsymbol{x}) \leq \boldsymbol{r}$ restore interconnection relations between currently selected or existing structural elements (corresponding $\left.y^{e}=1\right)$ and cancel relations for currently disappearing or nonexisting elements (corresponding $y^{e}=0$ ).

- Mixed linear constraints $\boldsymbol{K}^{e}+\boldsymbol{L}\left(\boldsymbol{d}^{c n}\right) \leq \boldsymbol{k}$ are proposed to define the continuous design variables for each existing structural element. The space is defined only when the corresponding structure element exists $\left(y^{e}=1\right)$, otherwise it is empty.

- Mixed linear constraints $\boldsymbol{P} \boldsymbol{y}+\boldsymbol{S}\left(\boldsymbol{d}^{\mathrm{st}}\right) \leq \boldsymbol{s}$ define standard design variables $\boldsymbol{d}^{s t}$. Each standard dimension $d^{\text {st }}$ is determined as a scalar product between its vector of standard dimension constants $\boldsymbol{q}$ and its vector of binary variables $\boldsymbol{y}^{s t}$. Only one discrete value can be selected for each standard dimension since:

$$
d^{\mathrm{st}}=\sum_{i \in I} q_{i} y_{i}^{\mathrm{st}} \quad \sum_{i \in I} y_{i}^{\mathrm{st}}=1
$$

\section{The optimization model}

The optimization model for a single-storey industrial building steel structure contains the mass objective function, (in)equality constraints and the variables (dimensions, internal forces, deflections, mass, etc.). Equality and inequality constraints represent a rigorous system of the design, loading, resistance, stress, deflections and stability functions. The dimensioning constraints are determined according to Eurocodes 3 (ultimate and serviceability limit states).

Resistance to bending moment of beams, columns and purlins:

$$
\begin{gathered}
M_{S d} \leq M_{e l, R d} \\
M_{e l, R d}=\frac{W_{e l} \cdot f_{y}}{\gamma_{M 0}}
\end{gathered}
$$

where $M_{S d}$ is the design bending moment, $M_{e l, R d}$ is the elastic resistance moment, $f_{y}$ is the yield strength of structural steel, $W_{e l}$ is the elastic section modulus and $\gamma_{M O}$ is the partial safety coefficient.

Resistance to axial force of the beams and columns:

$$
\begin{gathered}
N_{S d} \leq N_{p l, R d} \\
N_{p l, R d}=\frac{A \cdot f_{y}}{\gamma_{M 0}}
\end{gathered}
$$

where $N_{S d}$ is the design axial force, $N_{p l, R d}$ is the compression resistance, $A$ is the cross-section area, $\gamma_{M 0}$ is the partial safety coefficient.

Compression/buckling resistance of columns: 
648 High Performance Structures and Materials III

$$
\begin{gathered}
N_{S d} \leq N_{b, R d} \\
N_{b, R d}=\chi \frac{A \cdot f_{y}}{\gamma_{M 1}}
\end{gathered}
$$

where $N_{b, R d}$ is the compression/buckling resistances, $\chi$ is the reduction factors for the relevant buckling mode and $\gamma_{M I}$ is the partial safety coefficient.

Shear resistance of beams, columns and purlins:

$$
\begin{gathered}
V_{S d} \leq V_{p l, R d} \\
V_{p l, R d}=A_{v} \cdot \frac{f_{y}}{\sqrt{3}} \cdot \frac{1}{\gamma_{M 0}}
\end{gathered}
$$

where $V_{S d}$ is the design shear force, $V_{p l, R d}$ is the design shear resistance and $A_{v}$ is shear area.

Interaction between axial force and bending moment:

$$
\frac{N_{s d}}{N_{p l, R d}}+\frac{M_{s d}}{M_{e l, R d}} \leq 1.0
$$

Interaction between axial compression/buckling and bending moment lateraltorsional buckling:

$$
\frac{N_{s d}}{\chi \cdot A \cdot f_{y} / \gamma_{M 1}}+\frac{k_{L T} \cdot M_{s d}}{\chi_{L T} \cdot W_{e l} \cdot f_{y} / \gamma_{M 1}} \leq 1.0
$$

where $k_{L T}$ is the coefficient at lateral-torsional buckling, and $\chi_{L T}$ is the reduction factors for the relevant buckling mode at lateral-torsional buckling.

Inequality constraints of serviceability limit states for the vertical deflection of beams and purlins:

$$
\begin{array}{rlrl}
\delta_{\max , L} & \leq \frac{L}{200} & \delta_{\max , L f} \leq \frac{L_{f}}{200} \\
\delta_{2, L} \leq \frac{L}{250} & \delta_{2, L f} \leq \frac{L_{f}}{250}
\end{array}
$$

where $\delta_{\max . L}$ is the vertical deflection of beams and $\delta_{\max . L f}$ is the vertical deflection of purlins for the terminated state, $\delta_{2, L}$ is the vertical deflection of beams and $\delta_{2, L f}$ is the vertical deflection of purlins for the variable load. 
Inequality constraints of serviceability limit states for the horizontal deflections of portal frame:

$$
\Delta \leq \frac{H}{150}
$$

\section{The optimization}

The Modified Outer-Approximation/Equality-Relaxation (OA/ER) algorithm (Kravanja and Grossmann [6]) was used to perform the optimization. The $\mathrm{OA} / \mathrm{ER}$ algorithm consists of solving an alternative sequence of Non-linear Programming optimization subproblems (NLP) and Mixed-Integer Linear Programming master problems (MILP). The former corresponds to the optimization of parameters for a frame structure with fixed topology and standard dimensions and yields an upper bound to the objective to be minimized. The latter involves a global approximation to the superstructure of alternatives in which a new topology and standard sizes are identified so that its lower bound does not exceed the current best upper bound. The search is terminated when the predicted lower bound exceeds the upper bound.

The optimal solution of complex non-convex and non-linear MINLP problem with a high number of discrete decisions is in general very difficult to be obtained. The optimization is thus performed sequentially in two different phases to accelerate the convergence of the OA/ER algorithm. The optimization is proposed to start with the topology optimization of the frame, while standard dimensions are relaxed temporary into continuous parameters. When the optimal topology is found, standard sizes of cross-sections are re-established and the standard dimension optimization of beams, columns and purlins is then continued until the optimal solution is found.

\section{The example}

The paper presents an example of the topology and standard dimension optimization of a single-storey industrial building. The building is 30 meters wide, 50 meters long and 8 meters high, see Figure 2. The structure is consisted from equal non-sway steel portal frames which are mutually connected with the purlins.

The portal frame is subjected to self-weight $g$, uniformly distributed surface variable load $q$ (snow $s$ and wind $w$ ), concentrated variable load $P$ (wind $F$ and initial frame imperfection $F \varphi)$. Variable imposed load $\left(s=1.60 \mathrm{kN} / \mathrm{m}^{2}\right.$ and $w=$ $\left.0.137 \mathrm{kN} / \mathrm{m}^{2}\right)$ is defined as the uniformly distributed surface load in the model input data. Both, the horizontal concentrated load at the top of the columns and the vertical uniformly distributed line load on the beams are calculated considering the intermediate distance between the portal frames.

Design/dimensioning was performed in accordance with Eurocode 3. The design loads were calculated for the conditions of both ultimate limit states and serviceability limit states: (a) for ultimate limit states: $1.35 \cdot(g+s+w+F+F \varphi)$, 
(b): for serviceability limit states: $1.00 \cdot g+0.90 \cdot(s+w+F+F \varphi)$. While internal forces were calculated by the elastic first-order analysis the deformation of frame members were calculated by the force method.

The portal frame superstructure was generated in which all possible structures were embended by 30 portal alternatives, 20 purlin alternatives and different standard size variation. The superstructure also comprised 24 different standard hot rolled European wide flange I beams, i.e. HEA sections (from HEA 100 to HEA 1000) for each column, beam and purlin separately. The material used was steel S 355.

The optimization was performed by the MINLP optimization approach. The task of the optimization was to find the optimal structure mass, the optimal topology (the optimal number of portal frames and purlins) and optimal standard sizes.

The optimization was carried out by a user-friendly version of the MINLP computer package MIPSYN, the successor of PROSYN [6] and TOP [9]. As an interface for mathematical modelling and data inputs/outputs GAMS (General Algebraic Modelling System), a high level language, was used [10]. The Modified OA/ER algorithm and the two-phased optimization were applied, where GAMS/CONOPT2 (Generalized reduced-gradient method) [11] was used to solve NLP subproblems and GAMS/Cplex 7.0 (Branch and Bound) [12] was used to solve MILP master problems.

The optimization model contained 130 (in)equality constraints, 183 continuous and 122 binary variables. The final optimal solution of 150,87 tons was obtained in the $12^{\text {th }}$ main MINLP iteration.

The optimal result represents the mentioned optimal structure mass of 150,87 tons, the obtained optimal topology of 12 portal frames an 20 purlins (see Figure 2 ) and the calculated optimal standard sizes of columns, beams and purlins (see Figure 3).

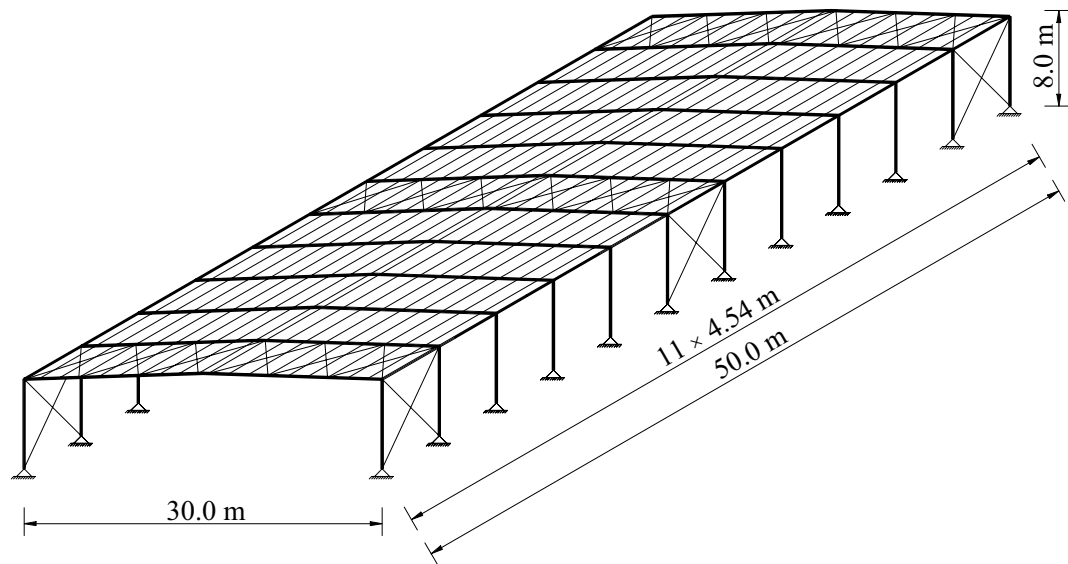

Figure 2: Optimal design of the single-storey industrial building. 


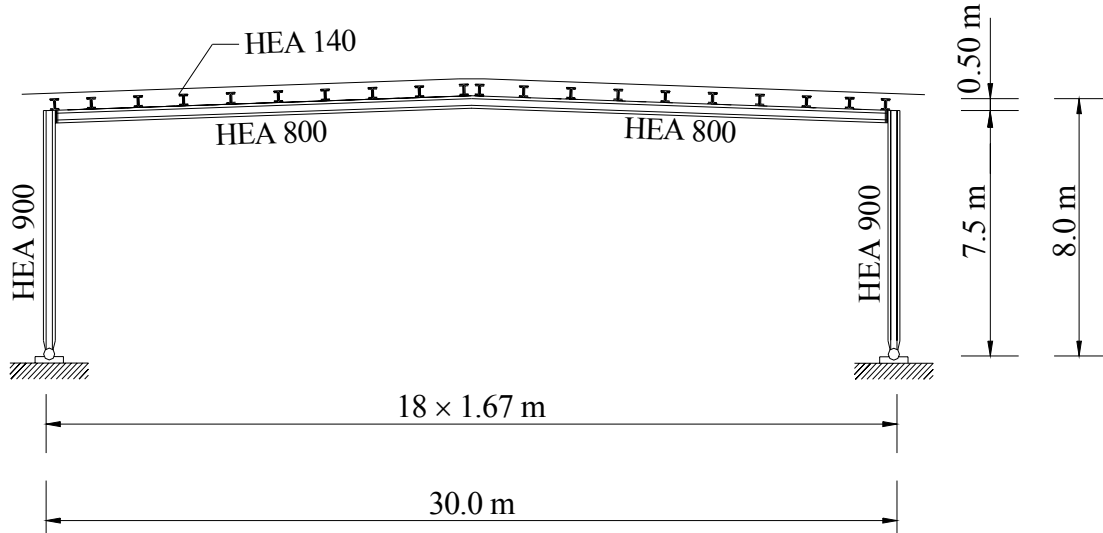

Figure 3: Optimal design of the portal frame.

\section{Conclusions}

The paper presents the topology and standard dimension optimization of the single-storey industrial building steel structure. The optimization is proposed to be performed by the Mixed-Integer Non-linear Programming (MINLP) approach. The MINLP was found to be a successful optimization technique for solving this type of structures.

\section{References}

[1] E.J. O'Brien, A.S. Dixon, Optimal plastic design of pitched roof frames for multiple loading, Comput. Struct. 64, 737-740, 1997.

[2] G. Gurlement, R. Targowski, W. Gutkowski, J. Zawidzka and J. Zawidzki, Discrete minimum weight design of steel structures using EC3 code, Struct. Multidisc. Optim. 22, 322-327, 2001.

[3] Eurocode 3, Design of steel structures, European Committee for Standardization, 1992.

[4] M.P. Saka, Optimum design of pitched roof steel frames with haunched rafters by genetic algorithm, Comput. Struct. 81, 1967-1978, 2003.

[5] S. Hernández, A.N. Fontán, J.C. Perezzán, P. Loscos, Design optimization of steel portal frames, Adv. Eng. Software. 36, 626-633, 2005.

[6] Kravanja, Z. and Grossmann, I.E., New Developments and Capabilities in PROSYN - An Automated Topology and Parameter Process Synthesizer, Computers chem. Eng., 18, 1097-1114, 1994.

[7] Kravanja, S., Kravanja, Z. and Bedenik, B.S., The MINLP optimization approach to structural synthesis. Part I: A general view on simultaneous topology and parameter optimization, Int. J. Numer. Methods Eng. 43, 263-292, 1998. 
[8] Kravanja, S., Kravanja, Z. and Bedenik, B.S., The MINLP optimization approach to structural synthesis. Part II: Simultaneous topology, parameter and standard dimension optimization by the use of the Linked two-phase MINLP strategy, Int. J. Numer. Methods Eng. 43, 293-328, 1998.

[9] Kravanja, S., Kravanja, Z., Bedenik, B.S. and Faith, S., Simultaneous Topology and Parameter Optimization of Mechanical Structures, Numerical Methods in Engineering '92, Proceedings of the First European Conference on Numerical Methods in Engineering, ed. Ch. Hirsch et al., pp. 487-495, Elsevier, Amsterdam, 1992.

[10] Brooke, A., Kendrick, D. and Meeraus, A., GAMS - A User's Guide, Scientific Press, Redwood City, CA, 1988.

[11] Drudd, A.S., CONOPT - A Large-Scale GRG Code, ORSA J. Comput. 6, 207-216, 1994.

[12] CPLEX User Notes, ILOG inc. 